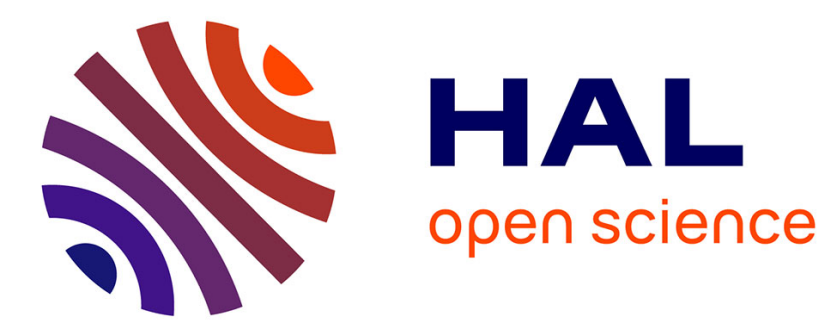

\title{
Definitions of Engagement in Human-Agent Interaction
}

Nadine Glas, Catherine Pelachaud

\section{To cite this version:}

Nadine Glas, Catherine Pelachaud. Definitions of Engagement in Human-Agent Interaction. International Workshop on Engagment in Human Computer Interaction (ENHANCE), Sep 2015, Xi'an, China. pp.944-949. hal-01213359

\section{HAL Id: hal-01213359 \\ https://hal.science/hal-01213359}

Submitted on 9 Oct 2015

HAL is a multi-disciplinary open access archive for the deposit and dissemination of scientific research documents, whether they are published or not. The documents may come from teaching and research institutions in France or abroad, or from public or private research centers.
L'archive ouverte pluridisciplinaire HAL, est destinée au dépôt et à la diffusion de documents scientifiques de niveau recherche, publiés ou non, émanant des établissements d'enseignement et de recherche français ou étrangers, des laboratoires publics ou privés. 


\section{Definitions of Engagement in Human-Agent Interaction}

\author{
Nadine Glas \\ Institut Mines-Télécom, Télécom ParisTech \\ CNRS, LTCI \\ Paris, France \\ Email: glas@telecom-paristech.fr
}

\author{
Catherine Pelachaud \\ CNRS, LTCI \\ Télécom ParisTech \\ Paris, France \\ Email: pelachaud@telecom-paristech.fr
}

\begin{abstract}
We give an overview of engagement in human-agent interaction. We discuss the different definitions of engagement in human and social science, specify how they relate to certain other concepts, and give an overview of the high level behaviour that is often associated with engagement. This work serves to position our future research on engagement in human-agent interaction.
\end{abstract}

Keywords—engagement; human-agent interaction; definitions

\section{INTRODUCTION}

Before working on aspects of engagement in human-agent interaction, such as the detection and synthesis of engagement, or strategies to favour the engagement level of the user, we need to know what engagement actually means in this domain. Over the last years different researchers have used different interpretations of the concept engagement leading to different definitions and meanings that coexist. In this work we try to give an overview of the interpretations of engagement in face-to-face human-agent interaction, thereby discussing the commonalities and differences between the definitions. We also shortly discuss concepts that are closely related to engagement and sometimes used interchangeably (Section IV). In this way we hope to clarify the existing terminology around engagement. Lastly we provide a concise overview of the types of high level behaviour that are associated with engagement and relevant in human-agent interaction (Section V).

\section{Engagement in Human-Agent Interaction}

In multiple human-agent studies the word engagement is used without providing an explicit definition of the term in that particular study (e.g. [1], [2], [3], [4], [5], [6]). For example [3] defines six levels of conversational engagement ranging from "no interest" to "governing/managing discussion" but without giving an explicit definition of what engagement actually is. In these cases we have to abstract the interpretation of the concept from the way in which the concept is used (measured/annotated/simulated/...), which makes that these interpretations are less straightforward to contribute to the understanding of the concept in general and to be reused in future studies.

In other studies, a clear definition of engagement has been given but exclusively for the purpose of that particular study. These definitions only cover the particular effect of engagement chosen to be measured in that study. [7] for example, expresses engagement in terms of spatial distance. In this work they characterize an engaged person as:

- "Mostly stationary near the booth, perhaps with a detected face." [7]

As such definitions are too context specific to serve as a description of the entire concept we no further elaborate on these. In the rest of this section we identify existing generic definitions of engagement in face-to-face human-agent interaction (including human-robot interaction) in order to obtain an overview of existing interpretations that could contribute to the understanding of the concept and the positioning of future engagement research. We do not discuss existing interpretations of engagement that could be used in the context of human-agent interaction, but limit this account to definitions that have actually been used in this context.

\section{A. Definitions}

Within face-to-face human-agent interaction there exists a range of different definitions of engagement. Some of them emphasize a particular point of view with a variation of this term such as face engagement and empathic engagement. In this section we identify all the pertinent definitions before categorizing their characteristics in table I and Section III.

In 1966 Goffman [8] formulated a definition of engagement that is still frequently used in human-robot interaction [16], [17]. Speaking about face engagements he claims that:

1) "Face engagements comprise all those instances of two or more participants in a situation joining each other openly in maintaining a single focus of cognitive and visual attention - what is sensed as a single mutual activity, entailing preferential communication rights." [8]

This interpretation is often used to describe the process through which people employ eye-contact, gaze and facial gestures to interact with each other [16].

Poggi (2007) [9] formulates engagement as:

2) "The value that a participant in an interaction attributes to the goal of being together with the other participant(s) and of continuing the interaction." [9]

This definition has been used for example in [18] (as user engagement), [19], [20], [21]. 
TABLE I. DECOMPOSED DEFINITIONS OF ENGAGEMENT.

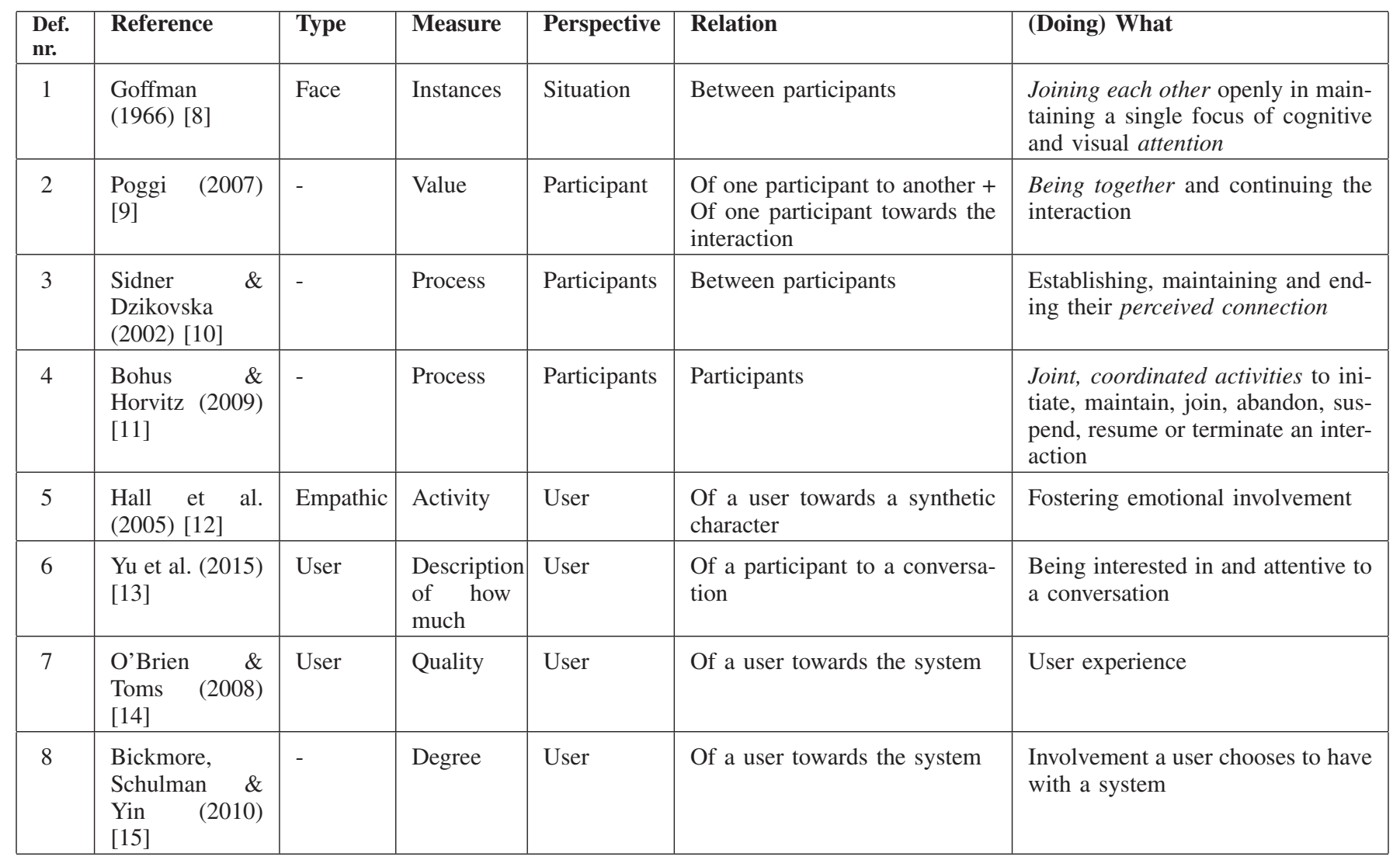

Another very frequently used definition (e.g. in [22], [23], [24]) comes from Sidner \& Dzikovska (2002) [10] who define engagement as:

3) "The process by which two (or more) participants establish, maintain and end their perceived connection. This process includes: initial contact, negotiating a collaboration, checking that other is still taking part in interaction, evaluating staying involved, and deciding when to end connection." [10]

A variation of this definition is formulated for the interaction model of Bohus \& Horvitz (2009) [11] where engagement is viewed as:

4) "The process subsuming the joint, coordinated activities by which participants initiate, maintain, join, abandon, suspend, resume or terminate an interaction." [25]

This interpretation in its turn is reused by [26].

Hall et al. (2005) [12] formulated a definition of empathic engagement:

5) "Empathic engagement is the fostering of emotional involvement intending to create a coherent cognitive and emotional experience which results in empathic relations between a user and a synthetic character." [12]

This definition differs with the former ones in the sense that it explicitly refers to the domain we are looking at by using the terms "user" and a "synthetic character" instead of the more generic term "participant".

Yu et al. (2004) [13] have defined a definition of engagement in the context of a voice communication system, that subsequently has been referred to in human-agent interaction [27]:

6) "User engagement describes how much a participant is interested in and attentive to a conversation." [13]

Some other interpretations of engagement are designed for the more general context of user-system interaction. In this context one often talks about user engagement. An important contribution to this term comes from O'Brien \& Toms (2008) [14] who have given an extensive overview of user engagement in this area. Their now widely used definition consists of the:

7) "Quality of user experience characterized by attributes of challenge, positive affect, endurability, aesthetic and sensory appeal, attention, feedback, variety/novelty, interactivity, and perceived user control." [14]

Lastly, Bickmore, Schulman \& Yin (2010) [15] consider engagement between users and machines over multiple interactions at once as:

8) "The degree of involvement a user chooses to have with a system over time." [15] 


\section{IMPLICATIONS}

Looking at the definitions in the previous Section, there are several commonalities and differences to notice. Table I summarises the characteristics of each definition (i.e. Def.).

\section{A. Connection and Cooperation}

First of all, an interaction requires a minimum level of connection and cooperation between its participants. Participants collaborate together in performing the joint action of interacting. It seems that in all above definitions engagement points to this cooperation and connection. In some this is made explicitly by speaking in terms of joining each other in, a goal of being together, a perceived connection and joint coordinated activities (see column 7, Table I). Others refer to a state that is the result of a connection and/or collaboration (e.g. emotional involvement, being interested in attentive to a conversation). In all the definitions the level of connection and cooperation is positively related to the presence or level of engagement.

\section{B. One- or Multi-Sided Relation}

While all definitions talk (indirectly) about a connection, the resulting relations differ. Some definitions imply a onesided established relation of a participant towards another participant or the interaction (Def. 2, 5-8), while other definitions consider the relations of all the interaction participants towards each other, implying a multi-sided relation (Def. 1, 3, 4).

\section{Participant or Interaction Perspective}

Another difference among the definitions relates to their perspectives. Sometimes engagement is attributed to the interaction, as in Def. 1 (instances in a situation). Sometimes engagement is seen as a concept that belongs to all the participants of the interaction (Def. 3,4). And other times it is attributed to a single participant (i.e. user) in the interaction (Def. 2, 5-8).

\section{Dynamic}

A commonality that we can derive from the above observations is that engagement is a concept which occurrence is dynamic. This is caused by the possibly constantly changing state of all the involved parties; the connection and relation between the interaction participants and/or the interaction itself can vary over time. Engagement thus evolves around a relation that is constantly influenced by (the participants of) the ongoing, continuously changing interaction.

\section{E. Implementation Measures}

There is no consensus on the measure of engagement. Some see it as an instance, others as a value or degree, a process, a description, etcetera (see Table I column 3). Measures like a value, a quality or instances describe engagement as a certain state (resulting from a connection and cooperation) that can be estimated at any moment in time, while other definitions focus on the process to get to this state. To this latter category belong Def. 3, 4 and 5 .

Within the cases where engagement relates to a state we can distinct two subcategories: those that assign a binary value to engagement (presence or absence, e.g. instance Def. 1) and those that imply a continuous notion of engagement (e.g. value Def. 2, degree Def. 8).

\section{F. Conclusion}

The different definitions all refer to a connection and relation of at least one participant in the interaction. The different perspectives and measurements ensure en emphasis on certain aspects over others. Depending on the goal of the study some definitions may be more suitable in certain circumstances than others. Studies may focus on a particular perspective, relation and measurement of engagement corresponding to a particular definition. For example, for some studies it may be interesting to consider the engagement of all participants at once (as in e.g. Def. 3 [10]) while for other studies it may be useful to focus on the engagement level of only one participant at a time (as in e.g. Def. 2 [9]). We would therefore like to argue in favour of multiple definitions coexisting.

\section{RElated CONCEPTS}

In the literature there are various concepts that are often mentioned in relation with engagement and that are sometimes used interchangeably [28] (as in Def 6). In this section we will demonstrate how exactly these concepts are related to engagement. We will discuss the terms attention, involvement, interest, immersion, rapport, empathy, and stance. We will give a global idea of the concepts to specify their relation to engagement without detailing all the coexisting definitions of the terms.

\section{A. Attention}

According to [28] in most studies relating to engagement two underlying fundamentals are apparent: attentional and emotional involvement. Attention is a vital aspect of engagement [19]. It controls the orientation of the senses towards stimuli that are of relevance to engagement [19]. Selective attention to a stimulus seems necessary for a basic form of engagement [28].

\section{B. Involvement}

[29] claims that involvement concerns being captured by the experience, an interpretation that [30] and [31] refer to as engagement. [19] states that cognitive and emotional involvement and commitment are key factors that underlie engagement. While the exact relation of involvement and engagement is thus disputable, it is clear that both concepts are closely related.

\section{Interest}

Interest can be seen as an emotional state that is related to the participant's goal of receiving and elaborating new and potentially useful knowledge [32]. Engagement is linked to and possibly caused by interest, and both interest and engagement are caused by attention [32]. According to [28] interest is often used to describe the motivation or goal towards opening and maintaining engagement. 


\section{Immersion}

Immersion is a term that is particularly in use in the area of virtual realities. It is often defined [33] as the experience of being transported to an elaborately simulated place or the sensation of being surrounded by a completely other reality [34]. Although there are many different interpretations of the term [35] immersion tends to refer to a broader concept than engagement. [35] claims in the context of game immersion that engagement can be seen as a part of immersion, namely as the first in three levels of immersion: engagement, moving on to greater involvement in engrossment and total immersion.

\section{E. Rapport}

[36] describes rapport in a broad manner as the feeling of being in sync with your conversational partner. [37] specifies that rapport only exists in interaction between individuals and that it consists of three essential components: mutual attentiveness, positivity and coordination. Engagement is also directly related to mutual attentiveness and coordination but not necessarily to positivity; one can be engaged and angry at the same time.

[38] designed a virtual agent to elicit rapport from human participants in a dyadic narrative task. They found that this agent produced increased speaker engagement [38], which shows a positive correlation between both concepts illustrated by simple contingent nonverbal behaviour. Such behaviour is employed in virtual agents to establish rapport with humans, but can also make agents more engaging [38].

\section{F. Empathy}

Empathy refers to the capability of sharing and interpreting correctly another's emotions and feelings [39]. It has been noted that we can distinguish two different kinds of empathy: a basic emotional contagion system where "one feels what the other feels" and a more advanced cognitive perspective-taking system where "one understands what the other feels" [40].

Central to both types of empathy is the sharing of one's feelings, which requires a connection between the participants. Engagement also implies a connection between participants. This makes that if a participant displays a high level of empathy (by for example mimicry [41], see section V-C) this person also appears to be engaged. The contrary is however not necessary. A connection between participants leading to engagement does not necessarily mean that the participants share or interpret each others' feelings; one can be highly engaged in a hostile discussion without empathizing with the interaction partner(s).

\section{G. Stance}

Stance is another term that is related to engagement and has many definitions. The overview of interpretations provided by [42] have led them to suggest that stance is "an attitude which, for some time, is expressed and sustained interactively in communication in a unimodal or multimodal manner". According to [43] a shared stance depends mainly on the ongoing interaction and depends as such on the engagement of the agents. [44] mentions that aggressive and provocative stances can be considered as special cases of engagement and disagreement.

\section{AsSOCIATED BEHAVIOUR}

Engagement is crucial in human-agent interaction as it is typically a prerequisite for the objective of the interaction: if a user stops interacting, the agent cannot have any further impact [15]. [10] states that conversation is impossible without engagement. It is thus important for agents to engage the user and maintain him/her engaged during the interaction. In this section we look at how engagement could manifest itself in human-agent interactions.

Agents can perform behaviour that might be associated with a certain level of engagement and their behaviour may influence the engagement of the other interaction participant(s) i.e. the user(s). This makes that for an agent there are two relevant tasks related to engagement: 1) interpreting the user's behaviour to infer a perceived level of engagement such that adequate reactions can be generated; and 2) demonstrating a certain level of engagement, which inevitably has an influence on the engagement of its user. For both of these tasks it is necessary to know how engagement can be expressed. In this section we summarise the types of high level behaviour that are most frequently associated with engagement in humanagent interaction. This high level behaviour can be performed by different types of low level behaviour. Low-level behaviour refers to actions as gestures, movements, gaze or speech where high-level behaviour refers to the goal or use of certain lowlevel behaviour in a particular situation.

\section{A. Synchrony}

According to the overview of [45] synchrony "refers to individuals' temporal coordination during social interactions". More specifically, it is "the dynamic and reciprocal adaptation of the temporal structure of behaviours between interactive partners" [45]. This is illustrated by an example of two people who cross or uncross their legs at the same time or gaze in the same direction simultaneously. The behaviours are multimodal as different (verbal and non-verbal) modalities intervene at the same time. But more important than the nature of the behaviours is the timing of the behaviours in comparison with the other participants' behaviours [45].

Synchrony has multiple effects in interaction between adults [45]. For instance, it contributes to smoothing social interactions and to achieve a coordination of expectancies among participants [46]. Most relevant to engagement is that synchrony fosters cooperation between individuals [47]. This shows that synchrony is related to engagement. [43] agrees that synchrony is a marker of mutual engagement.

\section{B. Alignment}

Very similar to synchrony is alignment. Alignment also expresses a coordination between interaction participants but this time at a deeper level. [48] refers to alignment as coordination that occurs when interlocutors share the same representation at some level [49], [50]. [48] has considered alignment at a linguistic level but for example [51] has demonstrated that the concept can be expanded to non-verbal behaviour. In contrast to synchrony which is multimodal, alignment always occurs in the same modality [45]. Just as synchrony, alignment is a measure of coordination which makes it a possible symptom of engagement. Besides, failure of alignment may cause failure 
to communicate successfully [52] influencing the participants' engagement directly.

\section{Mimicry}

Mimicry is behaviour displayed by an interaction participant who does what another person does and refers to an automatic tendency to imitate others [53]. Where in synchrony the important element is the timing, in mimicry the nature of the behaviour is important [45]. It contributes to the connection between the interaction participants, and favours empathy, liking and the smoothness of the interaction [41]. [53] agrees that mimicry enhances pro-social behaviour and helps to create bonds between individuals. Given that mimicry favours the connection between the participants it is another possible symptom of engagement.

\section{Feedback and Backchannels}

According to [54] several definitions of feedback have been proposed that all "agree on the fact that feedback strategies are used as a cooperative way of exchanging information about the successfulness of communication". This indicates that feedback is closely related to engagement.

Backchannels can be considered as a type of feedback [55]. Backchannels are "non-intrusive acoustic and visual signals provided by the listener during the speaker's turn" [56]. According to [32] backchannels provide information about the listeners engagement and processes which are necessary for communicative interaction: attention, perception, comprehension and internal reactions.

\section{E. Collaboration on a task}

Other behaviour that may be a cue for engagement can be found in the form of collaborating on a task. If participants cooperate and coordinate their actions they imply a perceived connection between them. [57] claims that engagement is supported by this ability to collaborate on a task, the use of conversation, and gestural behaviour.

\section{CONCLUSION}

We have given an overview of the different definitions of the term engagement in human-agent interaction and identified the differences and commonalities between the definitions. We further summarised to engagement related concepts and behaviour that are frequently mentioned in the context of human-agent interaction, with the goal of clarifying how the different concepts relate to each other. We will use this review for our future work on human-agent interaction where the agent's goal is to engage the user.

Given the number of definitions, the number of related concepts, and the range of behaviour that may be associated with engagement, we can state that engagement in humanagent interaction is a complex concept. In general, studies focus on a particular aspect of engagement without being able to cover the entire range of interpretations. We hope that future studies on engagement will take this complexity into account by explicitly circumscribing the aspects of engagement they tackle.

\section{ACKNOWLEDGEMENT}

This work was partially performed within the Labex SMART (ANR-11-LABX-65), supported by French state funds managed by the ANR, within the Investissements d'Avenir program under reference ANR-11-IDEX-0004-02. This work was also partially funded by the French project Avatar 1:1 and ANR MOCA.

\section{REFERENCES}

[1] C. Adam, L. Cavedon, and L. Padgham, "Hello emily, how are you today?: personalised dialogue in a toy to engage children," in Proceedings of the 2010 Workshop on Companionable Dialogue Systems. Association for Computational Linguistics, 2010, pp. 19-24.

[2] R. S. Baker, J. Ocumpaugh, S. M. Gowda, A. M. Kamarainen, and S. J. Metcalf, "Extending log-based affect detection to a multi-user virtual environment for science," in User Modeling, Adaptation, and Personalization. Springer, 2014, pp. 290-300.

[3] R. Bednarik, S. Eivazi, and M. Hradis, "Gaze and conversational engagement in multiparty video conversation: an annotation scheme and classification of high and low levels of engagement," in Proceedings of the 4th workshop on eye gaze in intelligent human machine interaction. ACM, 2012, p. 10.

[4] F. Bonin, R. Bock, and N. Campbell, "How do we react to context? annotation of individual and group engagement in a video corpus," in Privacy, Security, Risk and Trust (PASSAT), 2012 International Conference on and 2012 International Confernece on Social Computing (SocialCom). IEEE, 2012, pp. 899-903.

[5] I. Leite, C. Martinho, and A. Paiva, "Social robots for long-term interaction: a survey," International Journal of Social Robotics, vol. 5, no. 2, pp. 291-308, 2013.

[6] E. Mower, D. J. Feil-Seifer, M. J. Mataric, and S. Narayanan, "Investigating implicit cues for user state estimation in human-robot interaction using physiological measurements," in Robot and Human interactive Communication, 2007. RO-MAN 2007. The 16th IEEE International Symposium on. IEEE, 2007, pp. 1125-1130.

[7] M. P. Michalowski, S. Sabanovic, and R. Simmons, "A spatial model of engagement for a social robot," in 9th IEEE International Workshop on Advanced Motion Control. IEEE, 2006, pp. 762-767.

[8] E. Goffman, Behavior in public places: Notes on the social organization of gatherings. Simon and Schuster, 1966.

[9] I. Poggi, Mind, hands, face and body: a goal and belief view of multimodal communication. Weidler, 2007.

[10] C. L. Sidner and M. Dzikovska, "Human-robot interaction: Engagement between humans and robots for hosting activities," in Proceedings of the 4th IEEE International Conference on Multimodal Interfaces. IEEE Computer Society, 2002, p. 123.

[11] D. Bohus and E. Horvitz, "Models for multiparty engagement in openworld dialog," in Proceedings of the SIGDIAL 2009 Conference: The 10th Annual Meeting of the Special Interest Group on Discourse and Dialogue. Association for Computational Linguistics, 2009, pp. 225234.

[12] L. Hall, S. Woods, R. Aylett, L. Newall, and A. Paiva, "Achieving empathic engagement through affective interaction with synthetic characters," in Affective computing and intelligent interaction. Springer, 2005, pp. 731-738.

[13] C. Yu, P. M. Aoki, and A. Woodruff, "Detecting user engagement in everyday conversations," arXiv preprint cs/0410027, 2004.

[14] H. L. O'Brien and E. G. Toms, "What is user engagement? a conceptual framework for defining user engagement with technology," Journal of the American Society for Information Science and Technology, vol. 59, no. 6, pp. 938-955, 2008.

[15] T. Bickmore, D. Schulman, and L. Yin, "Maintaining engagement in long-term interventions with relational agents," Applied Artificial Intelligence, vol. 24, no. 6, pp. 648-666, 2010.

[16] J. Le Maitre and M. Chetouani, "Self-talk discrimination in humanrobot interaction situations for supporting social awareness," International Journal of Social Robotics, pp. 1-13, 2013. 
[17] A. Couture-Beil, R. T. Vaughan, and G. Mori, "Selecting and commanding individual robots in a vision-based multi-robot system," in Proceedings of the 5th ACM/IEEE international conference on Humanrobot interaction. IEEE Press, 2010, pp. 355-356.

[18] G. Castellano, A. Pereira, I. Leite, A. Paiva, and P. W. McOwan, "Detecting user engagement with a robot companion using task and social interaction-based features," in Proceedings of the 2009 international conference on Multimodal interfaces. ACM, 2009, pp. 119-126.

[19] C. Peters, C. Pelachaud, E. Bevacqua, M. Mancini, I. Poggi, and U. R. Tre, "Engagement capabilities for ecas," in AAMASO5 workshop Creating Bonds with ECAs, 2005.

[20] N. Glas and C. Pelachaud, "Politeness versus perceived engagement: an experimental study," in The 11th International Workshop on Natural Language Processing and Cognitive Science, 2014, pp. 135-148.

[21] J. Sanghvi, G. Castellano, I. Leite, A. Pereira, P. W. McOwan, and A. Paiva, "Automatic analysis of affective postures and body motion to detect engagement with a game companion," in Human-Robot Interaction (HRI), 2011 6th ACM/IEEE International Conference on. IEEE, 2011, pp. 305-311.

[22] G. Castellano, I. Leite, A. Pereira, C. Martinho, A. Paiva, and P. W. McOwan, "Detecting engagement in hri: An exploration of social and task-based context," in Privacy, Security, Risk and Trust (PASSAT), 2012 International Conference on and 2012 International Confernece on Social Computing (SocialCom). IEEE, 2012, pp. 421-428.

[23] A. Holroyd, C. Rich, C. L. Sidner, and B. Ponsler, "Generating connection events for human-robot collaboration," in RO-MAN, 2011 IEEE. IEEE, 2011, pp. 241-246.

[24] Y. I. Nakano and R. Ishii, "Estimating user's engagement from eyegaze behaviors in human-agent conversations," in Proceedings of the 15th international conference on Intelligent user interfaces. ACM, 2010, pp. 139-148.

[25] D. Bohus and E. Horvitz, "Learning to predict engagement with a spoken dialog system in open-world settings," in Proceedings of the SIGDIAL 2009 Conference: The 10th Annual Meeting of the Special Interest Group on Discourse and Dialogue. Association for Computational Linguistics, 2009, pp. 244-252.

[26] D. Klotz, J. Wienke, J. Peltason, B. Wrede, S. Wrede, V. Khalidov, and J.-M. Odobez, "Engagement-based multi-party dialog with a humanoid robot," in Proceedings of the SIGDIAL 2011 Conference. Association for Computational Linguistics, 2011, pp. 341-343.

[27] N. Novielli, F. de Rosis, and I. Mazzotta, "User attitude towards an embodied conversational agent: Effects of the interaction mode," Journal of Pragmatics, vol. 42, no. 9, pp. 2385-2397, 2010.

[28] C. Peters, G. Castellano, and S. de Freitas, "An exploration of user engagement in hci," in Proceedings of the International Workshop on Affective-Aware Virtual Agents and Social Robots. ACM, 2009, p. 9.

[29] C. L. Sidner, C. Lee, C. D. Kidd, N. Lesh, and C. Rich, "Explorations in engagement for humans and robots," Artificial Intelligence, vol. 166, no. 1, pp. 140-164, 2005.

[30] M. Lombard and T. Ditton, "At the heart of it all: The concept of presence," Journal of Computer-Mediated Communication, vol. 3, no. 2, pp. 0-0, 1997.

[31] M. Lombard, T. B. Ditton, D. Crane, B. Davis, G. Gil-Egui, K. Horvath, J. Rossman, and S. Park, "Measuring presence: A literaturebased approach to the development of a standardized paper-and-pencil instrument," in Third international workshop on presence, delft, the netherlands, vol. 240, 2000.

[32] C. Peters, C. Pelachaud, E. Bevacqua, M. Mancini, and I. Poggi, "A model of attention and interest using gaze behavior," in Intelligent Virtual Agents. Springer, 2005, pp. 229-240.

[33] A. McMahan, "Immersion, engagement and presence," The video game theory reader, pp. 67-86, 2003.

[34] J. H. Murray, Hamlet on the holodeck: The future of narrative in cyberspace. SimonandSchuster. com, 1997.

[35] E. Brown and P. Cairns, "A grounded investigation of game immersion," in CHI'04 extended abstracts on Human factors in computing systems. ACM, 2004, pp. 1297-1300.

[36] L. Huang, L.-P. Morency, and J. Gratch, "Virtual rapport 2.0," in Intelligent Virtual Agents. Springer, 2011, pp. 68-79.
[37] L. Tickle-Degnen and R. Rosenthal, "The nature of rapport and its nonverbal correlates," Psychological inquiry, vol. 1, no. 4, pp. 285293, 1990.

[38] J. Gratch, N. Wang, J. Gerten, E. Fast, and R. Duffy, "Creating rapport with virtual agents," in Intelligent Virtual Agents. Springer, 2007, pp. 125-138.

[39] J. Decety and P. L. Jackson, "The functional architecture of human empathy," Behavioral and cognitive neuroscience reviews, vol. 3, no. 2, pp. 71-100, 2004.

[40] S. G. Shamay-Tsoory, J. Aharon-Peretz, and D. Perry, "Two systems for empathy: a double dissociation between emotional and cognitive empathy in inferior frontal gyrus versus ventromedial prefrontal lesions," Brain, vol. 132, no. 3, pp. 617-627, 2009.

[41] T. L. Chartrand, W. W. Maddux, and J. L. Lakin, "Beyond the perception-behavior link: The ubiquitous utility and motivational moderators of nonconscious mimicry," The new unconscious, pp. 334-361, 2005.

[42] M. Chindamo, J. Allwood, and E. Ahlsen, "Some suggestions for the study of stance in communication," in Privacy, Security, Risk and Trust (PASSAT), 2012 International Conference on and 2012 International Confernece on Social Computing (SocialCom). IEEE, 2012, pp. 617622.

[43] K. Prepin, M. Ochs, and C. Pelachaud, "Mutual stance building in dyad of virtual agents: Smile alignment and synchronisation," in Privacy, Security, Risk and Trust (PASSAT), 2012 International Conference on and 2012 International Confernece on Social Computing (SocialCom). IEEE, 2012, pp. 938-943.

[44] J. Allwood, M. Chindamo, and E. Ahlsen, "On identifying conflict related stances in political debates," in Privacy, Security, Risk and Trust (PASSAT), 2012 International Conference on and 2012 International Confernece on Social Computing (SocialCom). IEEE, 2012, pp. 918925.

[45] E. Delaherche, M. Chetouani, A. Mahdhaoui, C. Saint-Georges, S. Viaux, and D. Cohen, "Interpersonal synchrony: A survey of evaluation methods across disciplines," Affective Computing, IEEE Transactions on, vol. 3, no. 3, pp. 349-365, 2012.

[46] A. Kendon, "Movement coordination in social interaction: Some examples described," Acta psychologica, vol. 32, pp. 101-125, 1970.

[47] S. S. Wiltermuth and C. Heath, "Synchrony and cooperation," Psychological Science, vol. 20, no. 1, pp. 1-5, 2009.

[48] M. J. Pickering, S. Garrod et al., "Toward a mechanistic psychology of dialogue," Behavioral and brain sciences, vol. 27, no. 2, pp. 169-189, 2004.

[49] H. P. Branigan, M. J. Pickering, and A. A. Cleland, "Syntactic coordination in dialogue," Cognition, vol. 75, no. 2, pp. B13-B25, 2000.

[50] S. Garrod and A. Anderson, "Saying what you mean in dialogue: A study in conceptual and semantic co-ordination," Cognition, vol. 27, no. 2, pp. 181-218, 1987.

[51] K. Bergmann and S. Kopp, "Gestural alignment in natural dialogue," in Proceedings of the 34th Annual Conference of the Cognitive Science Society (CogSci 2012), 2012.

[52] H. P. Branigan, M. J. Pickering, J. Pearson, and J. F. McLean, "Linguistic alignment between people and computers," Journal of Pragmatics, vol. 42, no. 9, pp. 2355-2368, 2010.

[53] R. B. Van Baaren, R. W. Holland, B. Steenaert, and A. van Knippenberg, "Mimicry for money: Behavioral consequences of imitation," Journal of Experimental Social Psychology, vol. 39, no. 4, pp. 393-398, 2003.

[54] J. Allwood and L. Cerrato, "A study of gestural feedback expressions," in First nordic symposium on multimodal communication. Copenhagen, 2003, pp. 7-22.

[55] S. Kopp, J. Allwood, K. Grammer, E. Ahlsen, and T. Stocksmeier, "Modeling embodied feedback with virtual humans," in Modeling communication with robots and virtual humans. Springer, 2008, pp. 18-37.

[56] V. H. Yngve, "On getting a word in edgewise," in Chicago Linguistics Society, 6th Meeting, 1970, pp. 567-578.

[57] C. L. Sidner, C. Lee, and C. Kidd, "Engagement during dialogues with robots," in AAAI spring symposia, 2005. 